\title{
Partial Discharge Signal Denoising by Discrete Wavelet Transformation
}

\author{
Trinurkalid Sumarwoto, ${ }^{\mathrm{a} *}$ Arief Basuki, ${ }^{\mathrm{b}}$ Umar Khayam, ${ }^{\mathrm{c}}$ Naohiro Hozumi, ${ }^{\mathrm{d}}$

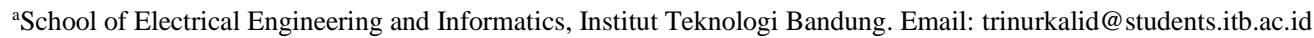

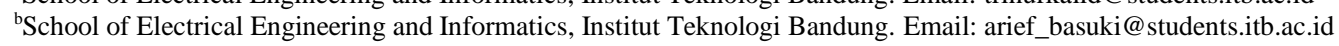 \\ ${ }^{c}$ School of Electrical Engineering and Informatics, Institut Teknologi Bandung. Email: umar@hv.ee.itb.ac.id \\ ${ }^{\mathrm{d} E l e c t r i c a l}$ and Electronic Information Engineering, Toyohashi University of Technology. Email: hozumi@ee.tut.ac.jp
}

\begin{abstract}
There are several techniques to eliminate noise on signals such as signal analysis in time zone, frequency area and short time Fourier Transform. As we know, frequency and time domain analysis are the basic methodologies in signal processing. In the partial discharge signal, the signal analysis can be realized in the time domain or in the frequency domain. When the signal is processed in the frequency domain, the time domain information becomes lost. To overcome these weaknesses, wavelet transformation is developed which can provide information in time and frequency domain. Wavelet transforms have been applied in various fields for signal processing in recent years. In this paper, a study of wavelet transformation was done to reduce noise from partial discharge signals obtained from previous experiment.
\end{abstract}

Keywords: Noise; partial discharge; threshold; wavelet

\section{Introduction}

According to IEC 60270 standard, partial discharge (PD) is a local electrification phenomenon that partially connecting the insulation between the conductors and occurs either on the surface of the conductor or in the insulation (void). This can occur in a conductor (conductor) in a short time, either at close range or at a great distance [1].

Many methods have been developed to detect PD signal. In the PD measurement, noise may arise from various sources that reduce the quality of measured data, consequently, the result signal is different to the PD signal. Fortunately, the noise may be removed. The technique for removing noise from PD signals can be realized in the time domain or in the frequency domain. PD and noise may be seen in different frequency characteristics [2]. However, when the signal is processed in the frequency domain, the time domain information becomes lost. To overcome these weaknesses, wavelet transformation is developed which can provide information in time and frequency domain. Wavelet transform (WT) has become a new and excellent tool for signal processing in recent years and has been successfully applied to various fields and applications [3].

*Corresponding author. Tel.:+81-90-266-05739

Toyohashi University of Technology

1-1 Hibarigaoka. Tempaku-cho. aichi. 441-8580, Japan
In this paper, a wavelet transformation is used to minimize noise contained inside PD signals which is obtained through previous experiment. The signal is already in the form of csv file from measurement through oscilloscope. The process of PD wavelet transformation is made using a matrix laboratory (MATLAB) program [4]. The method used in reducing PD signal noise in this study using wavelet transformation. The output of this application is a display of original PD signal that has not been filtered using wavelet, noise signal and signal that has been filtered through wavelet. In addition to measuring the level of noise, it also shows the value of Signal to Noise Ratio (SNR) to facilitate the analysis of wavelet transform (noise reduction) that has been done by the application.

\section{Signal Denoising Process}

\subsection{Partial Discharge (DP)}

Partial discharge occurs when the electric field inside the void exceeds the penetrating power of the gas in the void. When the AC voltage is given gradually then at a certain voltage value the partial discharge will begin to occur, this voltage is called the inception voltage (Vi). Where an inception voltage is the minimum voltage required for partial discharge to occur. When the voltage is raised and reaches 
the spark voltage, there will be no discharge until the initial avalanche electron begins to emerge partial discharge [5]. These electrons can arise due to the difference in the electric field in an area (electrons 1 in Fig. 1).

The electron avalanche

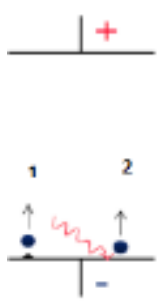

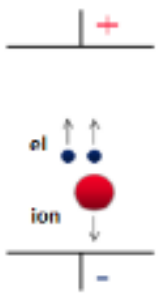

(a)

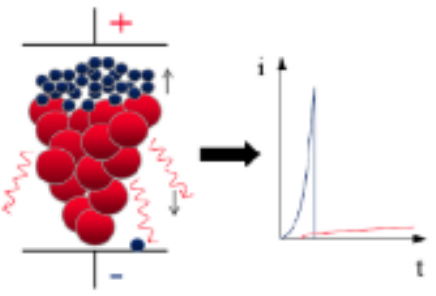

[b)
Figure 1. Discharge Process (a) avalanche electron and (b) discharge current [5]

\subsection{Wavelet Transformation}

Wavelet Transformation is a transformation that can provide resolution of frequency and time in the same time as well. There are two types of wavelet transformation, namely: Continyu Wavelet Transform (CWT) and Discrete Wavelet Transform (DWT), as follows :

\subsubsection{Continue Wavelet Transform (CWT)}

CWT works by calculating the convolution of a signal with a modulation window at any time with each desired scale. Modulation windows that have this flexible scale are called wavelets or wavelet base functions [9].

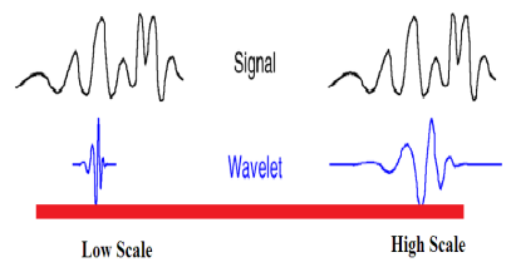

Figure 2. High-low scale relation with frequencyi [12]

In Fig. 2, we know that for low scale, wavelet signal undergoes compression and corresponds to higher frequencies. As for high-scale, the changes are roughly and slowly.

\subsubsection{Daubhecies Wavelet}

DWT is one of the wavelet transformation that represent signals in the time and frequency domain. The advantages of this DWT, easy to implement and efficient in terms of computing time. DWT is used to calculate the wavelet coefficients on all possible scales. DWT also generates enormous amount of data. Generally, the DWT process as follows : decomposition, filtering, downsampling and reconstruction [13].

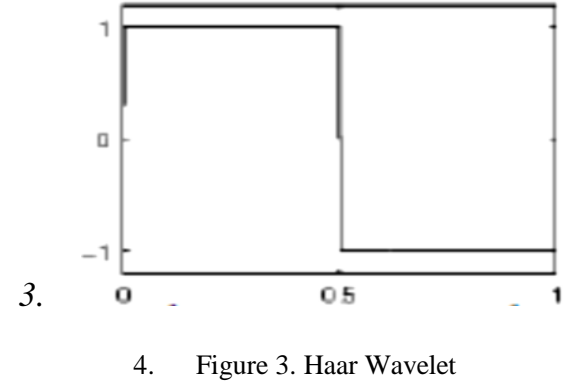

\subsection{Coiflet Wavelet}

\subsubsection{Haar Wavelet}

Haar Wavelet is the eldest and simplest wavelet. Haar Wavelet Haar is the same as Db1 Wavelet $\left(1^{\text {st }}\right.$ orde Daubechies) with the Haar Wavelet filter length is being 2 . $[4,12]$.

\subsubsection{Daubhecies Wavelet}

Daubhecies wavelet has a simple name, $\mathrm{Db}$., and for $\mathrm{N}$ orde is written with DbN. The Daubechies Wavelet Order is $\mathrm{N}=1$ or Haar, $\mathrm{N}=2, \ldots, \mathrm{N}=45$. Wavelet daubechies filter length is $2 \mathrm{~N}[4,12]$. We can see the Daubhecies wavelet in Fig. 6.
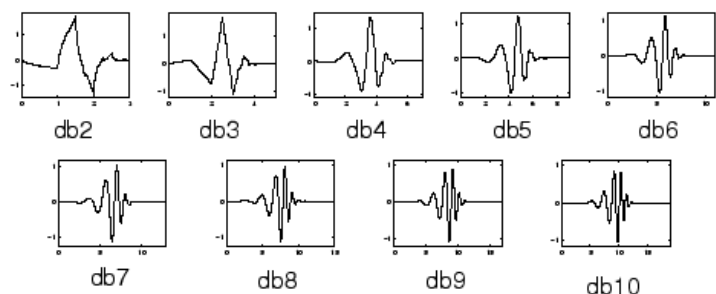

db5

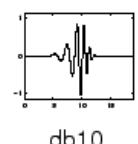

$\mathrm{db} 10$

Figure 4. Wavelet Daubhecies

\subsubsection{Coiflet Wavelet}

Wavelet Coiflet has a short name Coif, and for the order $\mathrm{N}$ is written with CoifN. The length of the Coiflet Wavelet filter is $6 \mathrm{~N}$ $[4,12]$. For its figure as attached below.

\subsubsection{Symlet Wavelet}

Wavelet Symlet has a sym short name, for the order $\mathrm{N}$ written with SymN. Wavelet Symlet has the order $\mathrm{N}=2$, $\ldots, 45$. The filter length for Wavelet Symlet is $2 \mathrm{~N}[4,12]$.

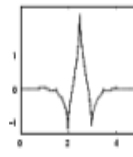

coif1

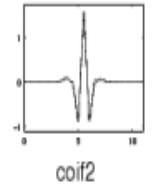

coif2

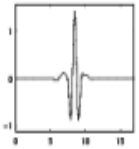

coif3

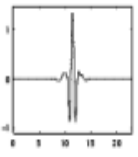

coif4

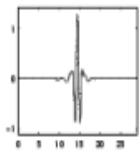

coif5
Figure 5. Wavelet Coiflet 

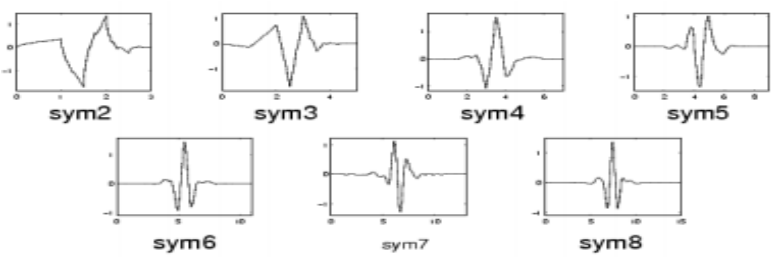

Figure 6. Symlet Wavelet

In this paper, we will use four types of wavelets namely, haar,daubhechies, symlet and coiflet. Although the haar is $1^{\text {st }}$ Daubhecies type and Symlet-Daubhecies are having the same length, we want to know form and SNR its signal.

Another purpose is the use of wavelets with the type of symlet and coiflet because this wavelet type has a different filter length so it can see which wavelet transformation is better, particulary for noise reduction.

\subsection{Thresholding [10]}

Threshold is a process that make zero for existing values within a predefined threshold value. Thresholding can be done globally where a threshold value is applied to all level of scale of the wavelet transform, or each scale level has its own threshold value. There are two threshold functions used in this task, as follow:

\subsubsection{Hard Treshold}

Hard threshold has an equation as follows :

$$
\delta_{\lambda}^{H}=\left\{\begin{array}{l}
\text { xif }|x|>\lambda \\
0 \text { if }|x| \leq \lambda
\end{array}\right.
$$

In the hard threshold there is a discontinuity in the threshold function so that the value of $x$ which has been mentioned before, above the threshold is not touched. At hard threshold only coefficients with absolute values below the threshold value are affected and the coefficients are replaced by zero whereas other than fixed.

\subsubsection{Soft Treshold}

$$
\delta_{\lambda}^{H}=\left\{\begin{array}{c}
x \text { if } x>\lambda \\
0 \text { if }|x| \leq \lambda \\
x+\lambda \text { if } x<\lambda
\end{array}\right.
$$

with $\lambda$ is threshold parameter.

The function of soft threshold is continue since $x$ value is above threshold. The use of soft threshold comes from the principle that noise affects the whole wavelet coefficient. At the soft threshold, all coefficients undergo a modification process. Coefficients with absolute values above the threshold value will be reduced in value whereas the other coefficients will be zero.

\subsection{Signal to Noise Ratio (SNR)}

Signal to Ratio (SNR) is a measure in determining the quality of a signal disturbed by noise. In other words SNR shows the comparison between the power of the original signal (information) with the noise. Here is the formula used in SNR calculation on this task [11]:

$$
S N R_{d B}=20 \log \frac{V s}{V n}
$$

With $\operatorname{SNR}_{\mathrm{dB}}$ (Signal Derau Ratio in Decibel unit), $V_{\mathrm{s}}$ (Signal Voltage), and $\mathrm{V}_{\mathrm{n}}$ (Noise Voltage).

\section{Measurement Method}

\subsection{Partial Discharge (PD) Measurement System}

Partial discharge measurement system that is used base on IEC 60270 standard. This detection method uses the recommended series of tests.

The main equipment which is used in Fig. 7: Voltage source $220 \mathrm{~V}$, transformator step-up $220 \mathrm{~V} / 100 \mathrm{kV}$, limiter resistor $6100 \Omega$, Coupling Capasitor $100 \mathrm{pF}$, Divider capacitor $68 \mathrm{nF}$ and Detecting impedance $50 \Omega$. .The discharges occurring in the sample are detected using the detection circuit High Frequency Current Transformer (HFCT), RC detector and Loop Antenna shown in fig. 7.

PD measurement result is given by oscilloscope that connect to Personal computer (PC). The PC is used to record and store test data in the form of images and CSV files. The test data is taken using Freeware software.

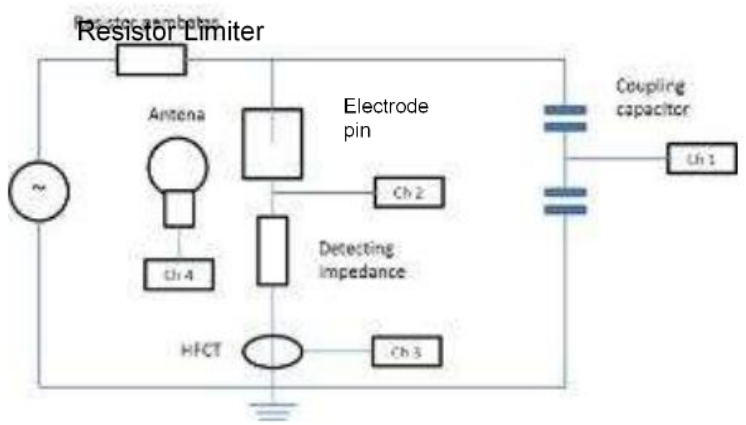

Figure 7. PD Measurement scheme [5]

After the PD data in CSV format has come out, it usually contain noise which cause difficult to make an analyze, the next step was denoising process by wavelet transformation.

\subsection{Wavelet Design}

This application was created using Graphical User Interface (GUI) Matrix Laboratory software (MATLAB) R2016a [1, 12]. The application which has been made, can reduce noise on the partial discharge signal by using the wavelet transform technique by passing signals on two different filter bands, then performing sub-sampling 
operation, then the signal is modified, and the last signal will be reconstructed.

In this program, to different output with one another and make it easy determining better one, it give SNR value before and after signal process. Figure 8 is the overall programming flowchart of this application :

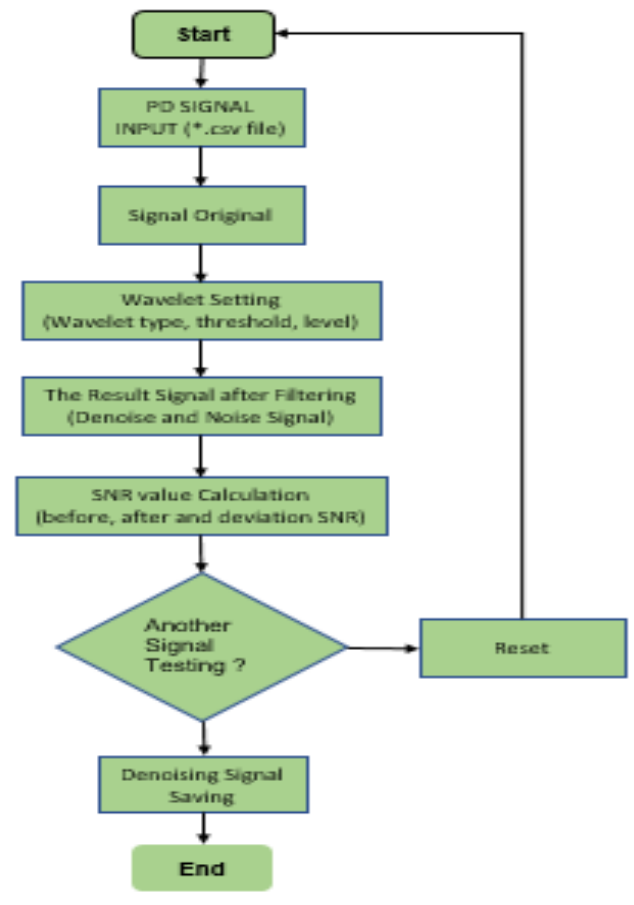

Figure 8. Programming Flowchart

\subsection{Process Analysis for Wavelet Design}

The first step is scalling. Volt/div is the value for each box on the oscilloscope in the vertical position. For each box on the oscilloscope will be written as many as 25 points in the CSV file. Therefore the $y$-axis value for each point on the CSV needs to be multiplied by the value of volt/div divided by 25 .

The next process is wavelet transformation process of PD signal with threshold parameters. There are 2 types of threshold used, namely soft and hard threshold. Uncorrect election of thresholding will result in less than maximum gain or noise generated.

After the process of selecting the type of threshold, the next process is selecting the type of wavelet process. There are four types of wavelets used in this task, haar, daubhecies, symlet and coiflet. For haar type, daubhecies type (db1,db2,db3,db4,db5,db6,db7,db8,db9,db10), the symlet type (sym2, sym3, sym4, sym5, sym6, sym7, sym8) and coiflet (coif1, coif2, coif3, coif4, coif5).

The fourth process is the selection of wavelet transformation levels consisting of levels 1 through 6 . After that we do denoising process. The noise signal itself is the difference between the original signal and signal after the wavelet process. Another important step is calculating the SNR value. It compares SNR before and after the wavelet transformation process.
The final step is storaging the result by the *.CSV form. Furthermore, this file can be used for judgment system or SNR evaluation. After analyzing one data, to make it easier to display other data, it can be done reset all values and display from previous data.

\section{Result and Discussion}

Consisting of application usage procedures, application testing, analysis through SNR values and graphical visualization of PD signals from practice results using a combination of three parameters (threshold, level and wavelet type). Because if it can be done, all combination of three parameters have 144 data, then in this report 144 data will be sampling for graphical visualization. For SNR value, it will be shown all number 144 data.

\subsection{Result [13]}

The test is done by simulating the combination of three wavelet parameters and comparing them visually and through the SNR value. The three wavelet parameters are: threshold, wavelet type and wavelet level.

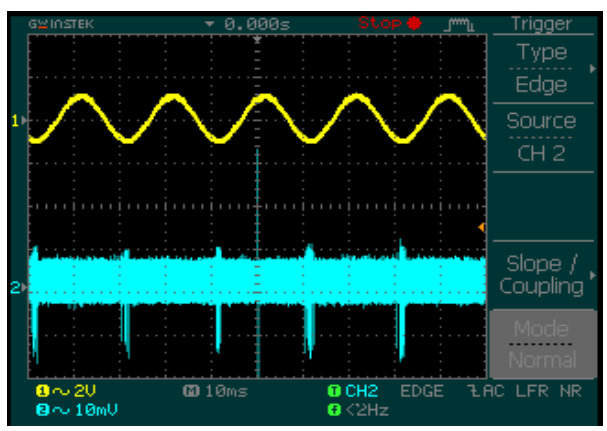

Figure 9. Positive PDIV on HFCT

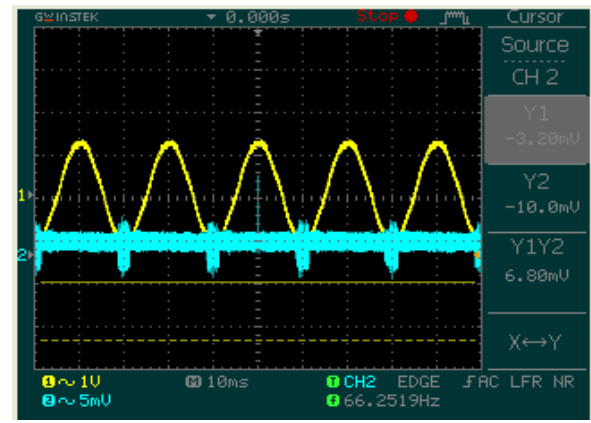

Figure 10. Positive PDIV on RC Detector

As a signal testing material, it will be used a partial discharge test signal by using the High Frequency Current Transformer (HFCT) and RC Detector [13]. The selection of this test signal at the time of partial discharge inception positive voltage (PDIV positive) as shown in Figs. 9 and 10.

The result of simulation test of partial discharge signal can be seen for HFCT processing signal with Hard Threshold, Level 6 and Coif5 wavelet type as shown Fig. 11 below: 


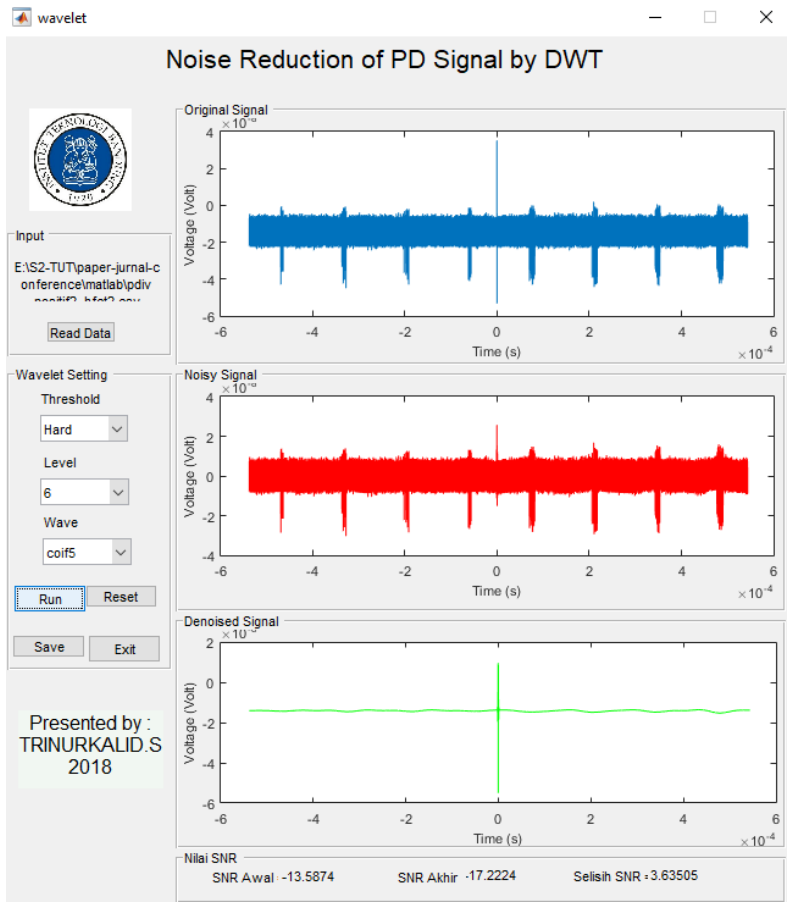

Figure 11. HFCT Denoising Signal Hard Threshold, level 6, coif5 wavelet type

For HFCT processing signal with Soft Threshold, Level 1 and Coif5 wavelet type as shown Fig. 12 below:

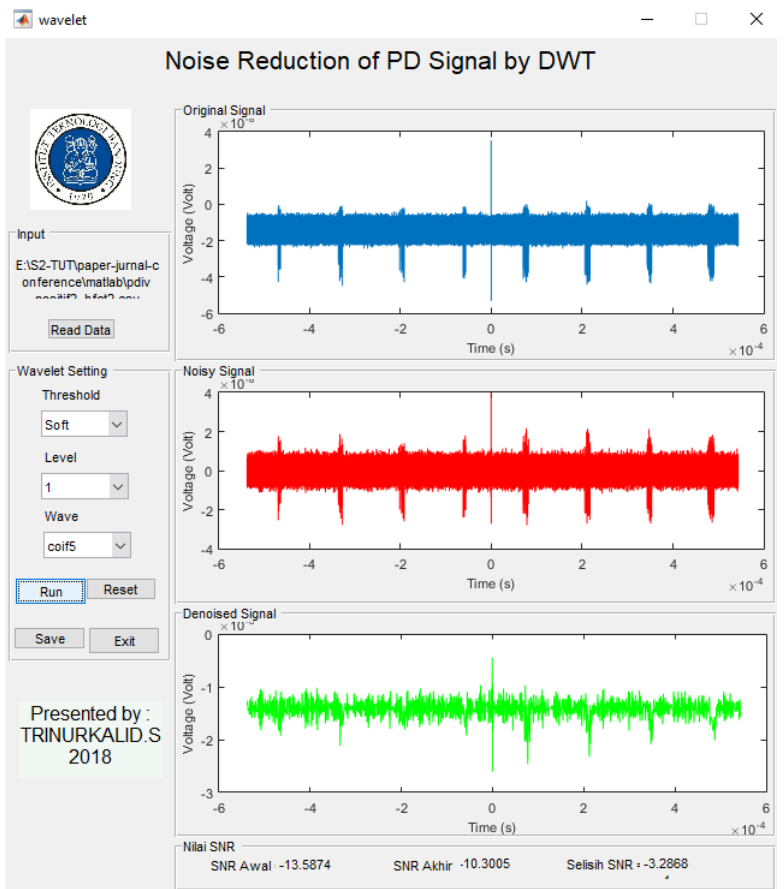

Figure 12. RC Detector Denoising Signal Hard Threshold, level 1, coif5 wavelet type

For HFCT processing signal with Soft Threshold, Level 6 and Coif5 wavelet type as shown Fig. 13 below:

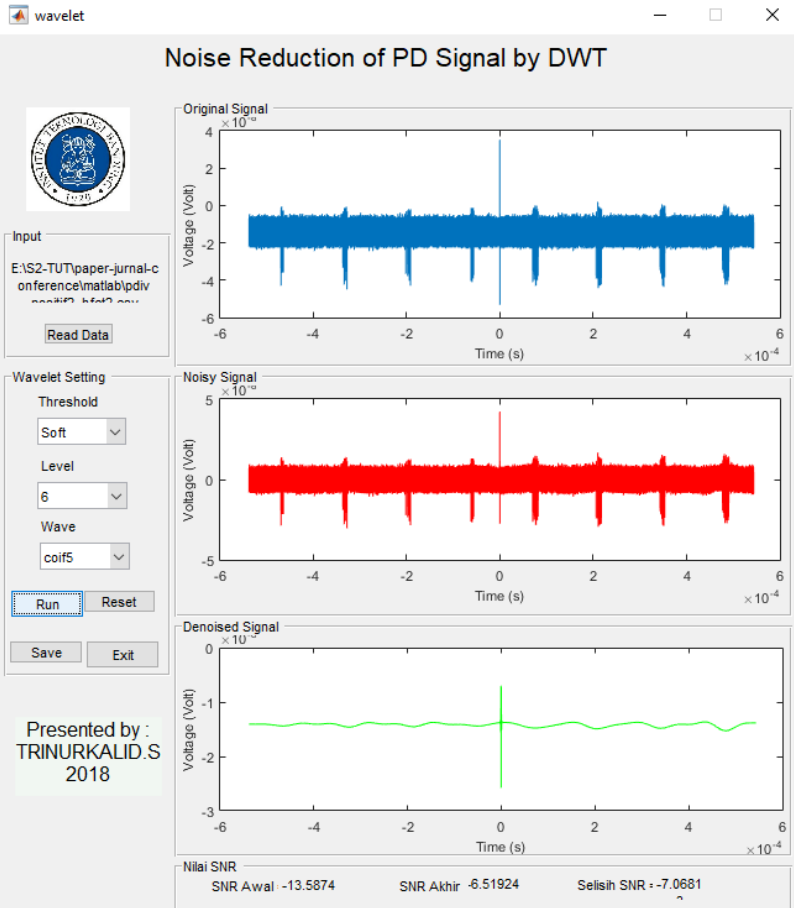

Figure 13. HFCT Denoising Signal Soft Threshold, level 6, coif5 wavelet type

By Fig. 11, we can see original signal, noisy signal and denoised signal with the result of first, last and SNR deviation are $-13.5874 \mathrm{~dB},-17.2224 \mathrm{~dB}$ and $3.63505 \mathrm{~dB}$. For Fig. 12, the result of denoising signal is $-13.5874 \mathrm{~dB}$, $10.3005 \mathrm{~dB}$ and $-3.2868 \mathrm{~dB}$. For Fig. 13, we could see the SNR value before, after and deviation : $-13.5874 \mathrm{~dB}$, $6.51924 \mathrm{~dB}$ and $-7.0681 \mathrm{Db}$.

For RC detector processing signal with Hard Threshold, Level 6 and Coif5 wavelet type as shown Fig. 14 below:

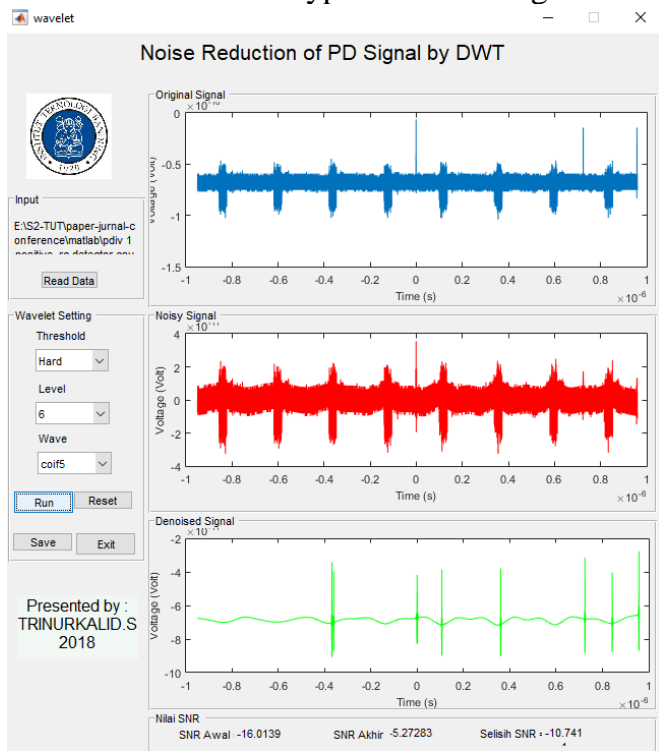

Figure 14. RC Detector Denoising Signal Hard Threshold, level 6, coif5 wavelet type 
For RC detector processing signal with Soft Threshold, Level 1 and Coif5 wavelet type as shown Fig. 15 below:

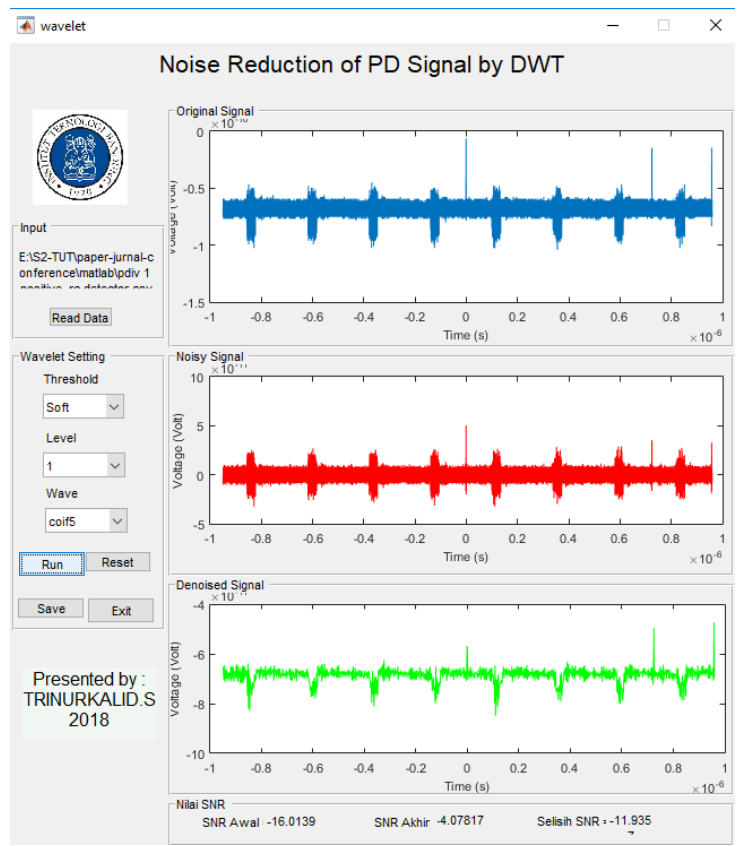

Figure 15. RC Detector Denoising Signal Hard Threshold, level 1, coif5 wavelet type

For RC detector processing signal with Soft Threshold, Level 6 and Coif5 wavelet type as shown Fig. 16 below:

By Fig. 14, like the result of HFCT, SNR in RC detector measurement give SNR as follow: $-16.0139 \mathrm{~dB},-5.27283 \mathrm{~dB}$ and $-10.741 \mathrm{~dB}$. If we change the threshold become soft and level become 1, as like Fig. 15, the SNR will be: -16.0139 $\mathrm{dB},-4.07817 \mathrm{~dB}$ and $-11.935 \mathrm{~dB}$.

Same as Fig. 15 but we change level to 6 , the SNR become: $-16.0139 \mathrm{~dB},-19.9331 \mathrm{~dB}$ and $-35.947 \mathrm{Db}$ as shown in Fig. 16.

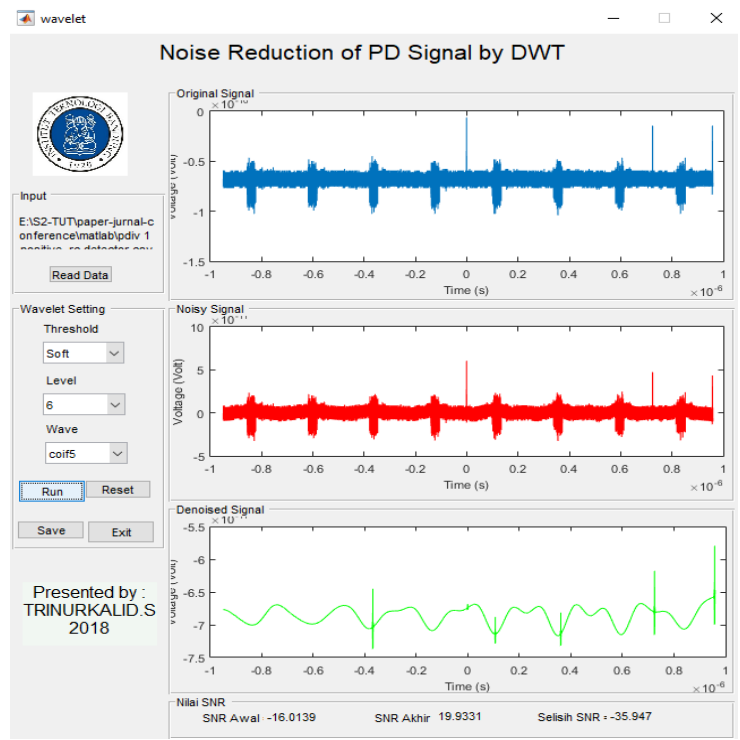

Figure 16. RC Detector Denoising Signal Soft Threshold, level 6, coif5 wavelet type

\subsection{Discussion}

From Figs. 11-13, the wavelet transformation simulation test result, threshold: hard; level: change; and wavelet type: coiflet5, we can get the following result:

Table 1. Test Result; type:coif5, threshold:hard

\begin{tabular}{|c|c|c|c|c|c|}
\hline \multirow{2}{*}{ Threshold } & \multirow{2}{*}{ Level } & \multirow{2}{*}{ Wavelet } & \multicolumn{3}{|c|}{ SNR } \\
\hline & & & before & after & Diff \\
\hline \multirow{6}{*}{ Hard } & 1 & \multirow{6}{*}{ Coif5 } & \multirow{6}{*}{-13.587} & -14.1579 & 0.5705 \\
\hline & 2 & & & -17.9043 & 17.9043 \\
\hline & 3 & & & -17.698 & 17.688 \\
\hline & 4 & & & -17.601 & 17.601 \\
\hline & 5 & & & -15.5385 & 15.5385 \\
\hline & 6 & & & -15.4951 & 15.4951 \\
\hline
\end{tabular}

Table 2. Test result; type:coif5, threshold:soft

\begin{tabular}{|c|c|c|c|c|c|}
\hline \multirow{2}{*}{ Threshold } & \multirow{2}{*}{ Level } & \multirow{2}{*}{ Wavelet } & \multicolumn{3}{|c|}{ SNR } \\
\hline & & & before & after & Diff \\
\hline \multirow{6}{*}{ Hard } & 1 & \multirow{6}{*}{ Coif5 } & \multirow{6}{*}{-13.587} & -10.3005 & -3.2869 \\
\hline & 2 & & & -10.4317 & 10.4317 \\
\hline & 3 & & & -9.13318 & 9.13318 \\
\hline & 4 & & & -8.36113 & 8.36113 \\
\hline & 5 & & & -7.14755 & 7.14755 \\
\hline & 6 & & & -6.51924 & 6.51924 \\
\hline
\end{tabular}

From the results shown in Tables 1 , it can be concluded that in the same wavelet type and threshold, generally, the higher the level of decomposition the better the value will be got. This can be seen from the increasing value of SNR after denoised and the smaller the difference.

Now, if we change the threshold to soft threshold like Figs. 12 and 13, we will see almost the same result like table1 but in more higher in final result after denoising process. The higher the decomposition level, the higher SNR will be got and the lower difference SNR will produced.

If we compare between Table 1 and 2 with the same level, it implies that the soft threshold give better result than hard threshold because it is cleared from Table 1 and 2, the SNR of soft threshold more higher than hard threshold SNR and also, by comparing the noised signal between Figs. 14 and 16 or Figs. 11 and 13, the amplitude signal of soft threshold more smaller than hard threshold so that soft threshold could eliminate the noise quite well.

Below the tabel summary for all wavelet parameter combination, Table 3 for HFCT and Table 4 for RD detector.

Table 3. HFCT of wavelet parameter combination

\begin{tabular}{cccccc}
\hline \multirow{2}{*}{ Threshold } & \multirow{2}{*}{ Level } & \multicolumn{2}{c}{ Wavelet type } & \multicolumn{2}{c}{ Result SNR (dB) } \\
\cline { 2 - 6 } & & Highest & Lowest & Highest & Lowest \\
\hline \multirow{5}{*}{ Hard } & 1 & coif1 & haar & -13.8665 & -19.2227 \\
\cline { 2 - 6 } & 2 & db6 & db2 & -15.8936 & -19.0412 \\
\cline { 2 - 6 } & 3 & db6 & haar & -15.7129 & -19.0143 \\
\cline { 2 - 6 } & 4 & db5 & haar & -16.5404 & -19.0315 \\
\cline { 2 - 6 } & 5 & db6 & haar & -15.5385 & -19.3231 \\
\cline { 2 - 6 } & 6 & db6 & coif1 & -15.4951 & -19.9518 \\
\hline \multirow{5}{*}{ Soft } & 1 & db8 & sym7 & -9.89927 & -12.8173 \\
\cline { 2 - 6 } & 2 & coif3 & db5 & -10.3567 & -12.063 \\
\cline { 2 - 6 } & 3 & db4 & db5 & -8.82599 & -10.5901 \\
\cline { 2 - 6 } & 4 & db4 & db5 & -7.58862 & -10.0539 \\
\cline { 2 - 6 } & 5 & db4 & db5 & -6.54698 & -9.3563 \\
\cline { 2 - 6 } & 6 & db4 & db5 & -6.51924 & -9.49774 \\
\hline
\end{tabular}


Table 4. RC detector of wavelet parameter combination

\begin{tabular}{cccccc}
\hline \multirow{2}{*}{ Threshold } & \multirow{2}{*}{ Level } & \multicolumn{2}{c}{ Wavelet type } & \multicolumn{2}{c}{ Result SNR (dB) } \\
\cline { 2 - 6 } & & Highest & Lowest & Highest & Lowest \\
\hline \multirow{5}{*}{ Hard } & 1 & sym6 & db6 & -6.20452 & -11.6479 \\
\cline { 2 - 6 } & 2 & sym6 & db6 & -2.83113 & -10.9276 \\
\cline { 2 - 6 } & 3 & sym6 & db6 & -2.03524 & -10.721 \\
\cline { 2 - 6 } & 4 & sym6 & sym7 & -1.31779 & -10.6421 \\
\cline { 2 - 6 } & 5 & sym6 & db6 & -1.20826 & -10.6117 \\
\cline { 2 - 6 } & 6 & sym6 & db6 & -1.21057 & -10.852 \\
\hline \multirow{5}{*}{ Soft } & 1 & haar & sym3 & -0.82448 & -6.6141 \\
\cline { 2 - 6 } & 2 & haar & db2 & 2.30468 & -1.46216 \\
\cline { 2 - 6 } & 3 & coif4 & db2 & 6.85471 & 2.11982 \\
\cline { 2 - 6 } & 4 & sym8 & sym2 & 14.8486 & 3.23104 \\
\cline { 2 - 6 } & 5 & sym4 & sym2 & 20.7808 & 3.7416 \\
\cline { 2 - 6 } & 6 & sym6 & db2 & 31.8172 & 1.87374 \\
\hline
\end{tabular}

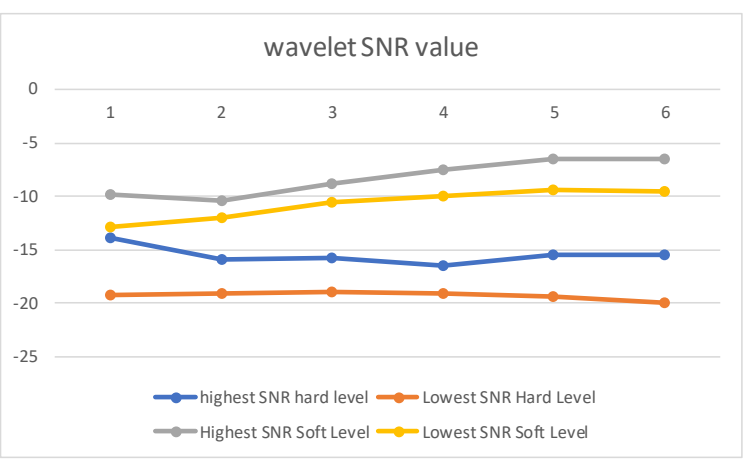

Figure 17. Comparison of HFCT SNR values

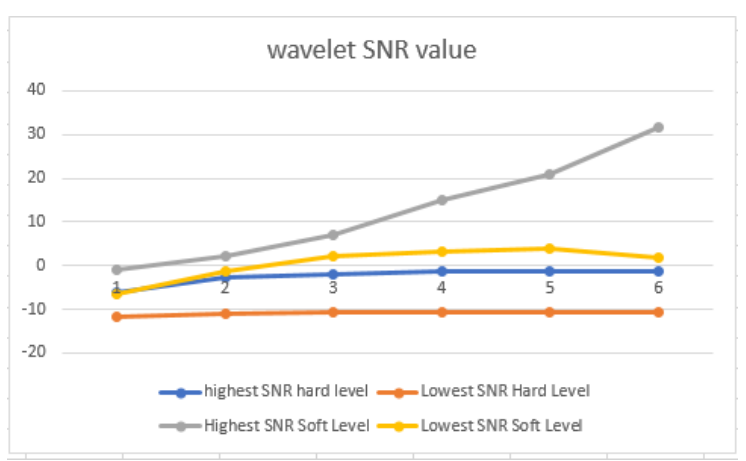

Figure 18. Comparison of RC detector SNR values

By Table 3 and 4, it can be seen that SNR signal increases inline with its level, for soft mode more better than hard mode. The highest SNR value, for table-3, at level 6 with wavelet Daubhecies-4 type, for soft threshold $=-6.51924 \mathrm{~dB}$ and wavelet Daubhecies-6, for hard threshold $=-15.4951 \mathrm{~dB}$ and for Table 4, with symlet- 6 type for soft threshold = $31.8172 \mathrm{~dB}$ and hard threshold $=-15.4951 \mathrm{~dB}$ This indicates that in soft mode there is more noise reduction than hard.

By Figs. 17 and 18, it can be seen visually that at each increase of wavelet level, more noise will be eliminated. This could be happened, because soft threshold is more sensitive than hard threshold. The soft threshold uses three equations to denoise the partial discharge signal, but the hard threshold only use two equations [4].

\section{Conclusion}

It is usually not easy to analyze partial discharge signal directly because noise comes from various sources that can reduce the quality of data received, consequently, the result signal which is obatained did not equal to the expectation. By the wavelet system, the noise could be reduced significantly.

In this system soft threshold mode is better than hard threshold mode for reduction noise and the higher level parameter was used in same level, the better signal can be analyzed for next pace. It happened because the soft threshold uses three equations to denoise the partial discharge signal, but the hard threshold only use two equations.

\section{Acknowledgements}

We acknowledge PLN for the financial support during study and research stay of Trinurkalid Sumarwoto in High Current and Voltage Laboratory, Bandung Institute of Technology, Bandung, Indonesia.

\section{References}

[1] IEC 60270,2000, "Hight Voltage Test Technique - Partial discharge measurement"

[2] Xiteng Liu, "A New Approach to Time-Frequency Analysis". IEEE Data Compression Conference, pp. 540, 2010.

[3] Vidya H. A, V. Krishnan and Mallikarjunappa K, "A Wavelet Transform Technique for De-noising Partial Discharge Signals", IEEE Int. Conft. nn Condition Monitoring and Diagnosis, Beijing, China, April 21-24, 2008.

[4] Tria Kasnalestari, " Wavelet Transformation Application For Denoising Sinyal Partial Discharge", Bandung Institute of Technology, 2016

[5] Suwarno, "Diagnosis of High Voltage Equipments", ITB Press, 2010.

[6] Emeraldi Primas, " Engineering and Implementation of Impedance Adjustment Circuit For Partial Discharge Measurement In Frequency Range To One Giga Herzt"Thesis of Postgraduate Program, High Voltage Enegineering, STEI, ITB, Bandung, 2013.

[7] Umar Khayam,"Partial Discharge Experiment Module." High Voltage Laboratory of ITB, 2014.

[8] Ibrahim Alhanif, " Preparation of Partial Discharge Detection and Measurement ", Thesis of Postgraduate Program, High Voltage Enegineering, STEI, ITB, Bandung, 2015.

[9] Rendi Pradila, 2013, Introduction of Wavelet, term paper seismology resume, Brawijaya University.

[10] Alok Kumar P.,’Analysis of Partial Discharge Signals Using Digital Signal Processing Techniques", Natonal Institute of Technology Rourkla, 2012.

[11] Hilton Mota,"Paritial Discharge Signal ProcessingUsing Wavelet", Univesulde Federal de Sao Joao del Rei, Bazil 2010.

[12] Swastiti Vinana Sari, Achmad Hidayatno, Abdul Syakur, "Wavelet Transformation Application To Eliminate Noise On Partial Partition Signals", Electrical Engineering, Diponegoro University , Semarang, Indonesia, 2008

[13] Group 8,"Modul II-Partial Discharge", Laboratory Report, High Voltage Laboratory, STIE-ITB, 2016 